\title{
Polarization properties of luminescence from silicon nanocrystals
}

\author{
J. Diener, D. Kovalev, G. Polisski, F. Koch \\ Technische Universität München, Physik-Department E16, 85747 Garching, Germany
}

\begin{abstract}
Polarization dependent photoluminescence (PL), time-resolved PL and PL excitation experiments are performed in order to clarify the origin of the linear polarization of the PL of porous silicon excited by linear polarized light. It is shown that this effect, when PL is excited significantly above the detection energy, is not related to a coherent exciton alignment or selective optical excitation of those nanocrystals whose transition dipole moments are oriented parallel to the polarization vector of the exciting light. The experimental results are interpreted in the framework of a dielectric model assuming aspheric nanocrystals.
\end{abstract}

Keywords: porous silicon, silicon nanocrystals, polarized photoluminescence

Paper received 24.07.00; revised manuscript received 05.09.00; accepted for publication 12.12.00.

Recent years most of the optical studies of silicon $(\mathrm{Si})$ nanocrystals (NCris) have been concentrated on quantum confinement modifications of their absorbing and emitting states [1]. Another important consequences of the reduced dimensionality arising from the nonspherisity of the $\mathrm{Si}$ NCŕs in porous $\mathrm{Si}$ (PSi) are optical effects due to dielectric nano-structuring of bulk Si. The most prominent onse are the linear polarization memory phenomena observed in the visible photoluminescence (PL) from Si NCrs in PSi $[2,3]$. For bulk semiconductors polarization resolved PL measurements are widely used to investigate the electronic symmetry of the luminescing state and energy relaxation processes. Polarized PL can be a result of crystalline lattice structure or external perturbations. In order to observe polarized light emission from a mutlivalley cubic semiconductor like $\mathrm{Si}$ it is necessary to lift the degeneracy of the hole band or valleys, respectively, by an external uniaxial perturbation [4]. Excitation of the PL by linear or circular polarized light selects an individual states among spin-, angular momentum-, or linear momentum-degenerated states and if their relaxation is absent results in a polarized PL [5]. Low dimensional structures exhibit PL polarization as well since quantum confinement effects can be regarded as a strong perturbation that lowers the crystal symmetry.

Besides electronic contribution to the polarization of the PL the modification of the electric field via dielectric screening effects has to be taken into account. For quantum well structures this effect is a minor one since dielectric constants of the layers are almost the same. On the contrary, the lateral modulation of the dielectric con- stant in nanocrystalline assemblies can be fairly large due to a large difference between the dielectric constants of the nanocrystals and the surrounding [6]. Therefore both, the electric field and the dipole moment of the interband transitions, contribute to the polarization of the light emission from nanocrystalline assemblies.

PSi exhibits strongly linearly polarized PL when excited with linear polarized light $[2,3]$. The strength of this effect is expressed by the degree of polarization $\rho=\left(I_{\|}-\right.$ $\left.-I_{\perp}\right) /\left(I_{\|}+I_{\perp}\right)$ where $I_{\|}\left(I_{\perp}\right)$ are the intensities of the PL polarized parallel (perpendicular) to the direction of the electric field of the exciting light. Fig. 1a shows the visible PL band excited at $2.8 \mathrm{eV}$ and the general spectral behavior of $\rho$. $\rho$ is positive over the whole visible spectral range. Contrary to that the near infrared PL band, observed at low temperatures (Fig. 1b), is found to be completely unpolarized. The polarization of the PL vanishes at the bandgap of bulk Si. Because the near infrared emission band is due to point defect luminescence (dangling bond states on the surface of the NCrs), the large value of $\rho$ for the visible PL is clearly linked to intrinsic optical properties of the Si NCrs.

However, this PL polarization phenomenon can not be understood in traditional terms like exciton alignment or selective optical excitation of those nanocrystals whose transition dipole moments are oriented parallel to the polarization vector of the exciting light. The nature of the absorbing and emitting dipoles has to be quite different because, as can be seen in Fig. 1 a, the photoexcited $e-h$ pair looses an energy on the order of an $\mathrm{eV}$ in inelastic relaxation processes and therefore the observed PL po- 


\section{J. Diener et al.: Polarization properties of luminescence from silicon ...}

a)
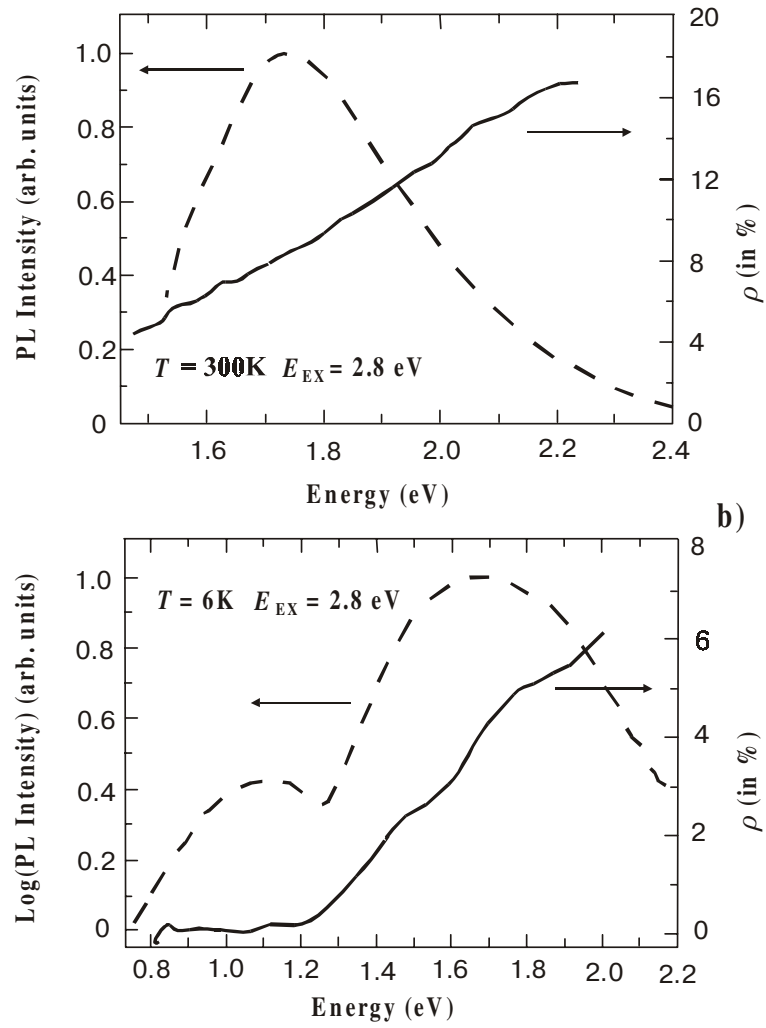

Fig. 1. The PL spectra (dashed lines) and the corresponding $\rho$ (line) for two different standard PSi samples at $300 \mathrm{~K}$ (a) and $6 \mathrm{~K}$ (b).

larization is not a coherent effect. Another confirmation of this incoherence can be deduced from polarized photoluminescence excitation (PLE) experiments. For the PLE experiment the detection energy is fixed at $2 \mathrm{eV}$ and the energy of the linearly polarized exciting light is varied. The PL response is normalized to the intensity of incident light. The orientation of the polarization vector of the detected light is defined by a polaroid placed in the detection path. PL is excited and detected in normal to the sample surface incidence/PL collection geometry. Fig. 2 depicts the intensities of the PL polarized parallel and perpendicular to the polarization of incident light as a function of its energy. If PL is excited in parallel polarization conditions its intensity is always higher than that in the perpendicular polarization configuration. The resulting $\rho$ is shown in the inset of Fig. 2. Over the whole energy range, from $2.2 \mathrm{eV}$ to $2.8 \mathrm{eV}$, no distinct variation of the degree of linear polarization is observed. For other nanocrystalline systems like CdSe NCrs polarized PL is only observed under resonant excitation conditions where the energy relaxation of the carriers is small and, therefore, the memory of the initial dipole orientation is conserved [7]. Contrary to that in PSi the level of the PL polarization is energy independent.

Even in the absence of energy relaxation the initial electronic dipole orientation can be scrambled if the lifetime of optical transition is long enough. For instance,

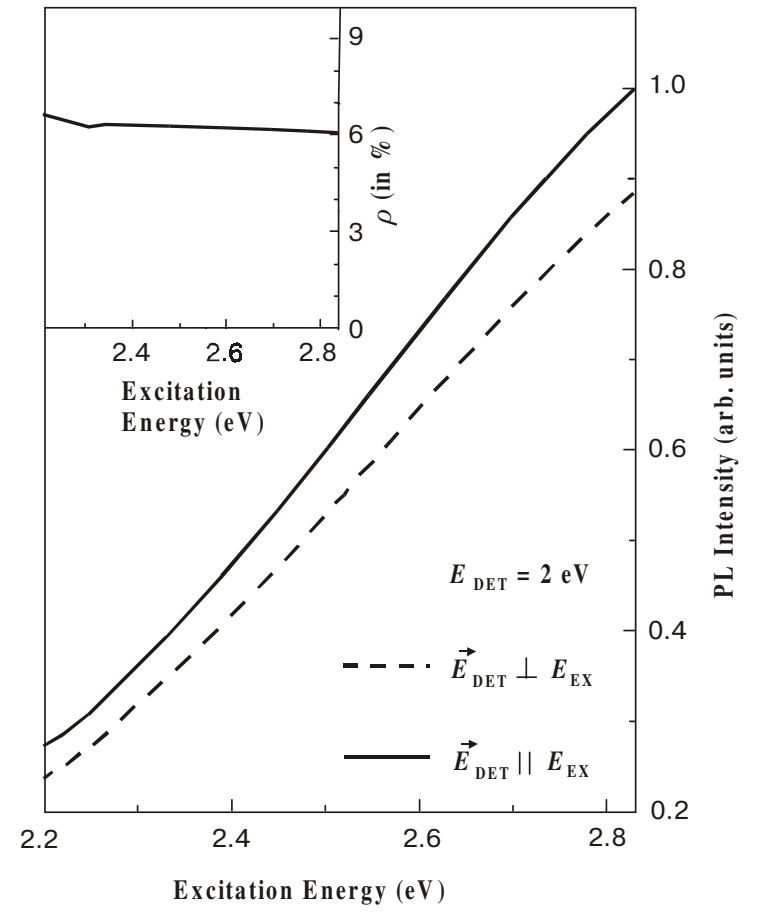

Fig. 2. Polarization resolved PLE spectra. The inset shows the calculated $\rho$.

high positive values of $\rho$ have been observed only at the very early times of the PL decay (nanosecond time domain) [8]. Fig. 3 depicts the degree of linear polarization versus time for different detection energies and for the integrated PL signal. PL is excited by a linear polarized pulsed laser $(3.67 \mathrm{eV}, 300 \mathrm{ps})$ and its decay is measured for parallel and perpendicular polarized PL components. We found that the value of linear polarization of the PL from PSi does not depend on time within a microsecondmillisecond time scale.

Therefore, this unusual behavior of polarized PL from PSi can not be attributed to an alignment of the excited transition dipole. Taking into account that other Si based nanocrystalline materials like $\mathrm{Si} \mathrm{NC}$ rs prepared by $\mathrm{Si}$ implantation in $\mathrm{SiO}_{2}$ layers or by chemical synthesis, while having the same general luminescence properties, do not show this effect, we conclude that the shape of nanocrystals rather than quantum confinement effects seems to be the major reason for this phenomenon. Nonspherical NCirs will preferentially absorb and emit light which is polarized parallel to their long axis. Since all dimensions of the $\mathrm{NC}$ are smaller than the wavelength of light this problem can be solved in classical electrostatic terms. The electric field of the light induces polarization charges at the surface of the NC. These charges create a depolarization field that decreases the strength of the electric field inside the NC. This effect is weaker if the 


\section{J. Diener et al.: Polarization properties of luminescence from silicon ...}

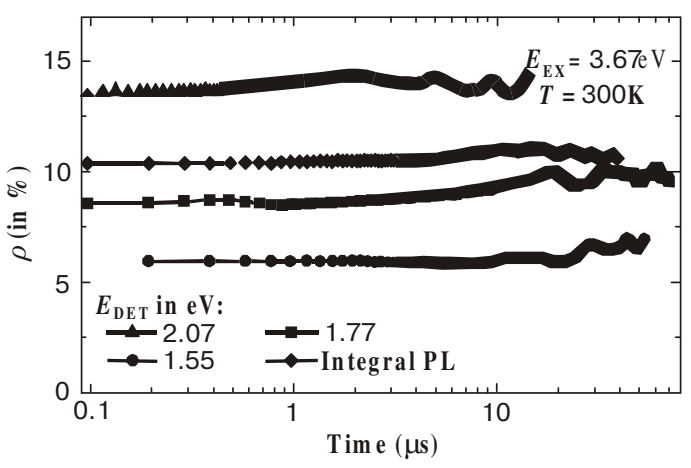

Fig. 3. $\rho$ as a function of time at different detection energies.

electric field is applied along the long axis of the NC. The probability of optical absorption is proportional to the square of the electric field inside the NC and therefore NCrs with their long axes aligned in the direction of the light polarization will preferentially absorb light. The probability of spontaneous emission is proportional to the square of the electric field of a photon inside the NC. This field is again largest for photons with their polarization vector along the large dimension of the $\mathrm{NC}$.
Therefore the emission is also preferentially linearly polarized. The strength of this effect is determined by the shape of the NC and by the difference between the dielectric constants of nanocrystals and the surrounding matrix [9]. However, these arguments refer to an individual $\mathrm{NC}$ with given aspheric shape while the PL and its polarization level are average characteristics of nanocrystalline assemblies. Therefore, in order to explain the polarization effects in the PL of PSi, the overall space distribution of differently shaped NCrs has to be taken into account. The importance of that can be demonstrated in an experiment where $\rho$ is referred to the in-plane rotating polarization direction of the exciting light. Under normal incidence of the exciting light on (100) PSi layer (parallel to the growth direction), the PL is always partially linearly polarized, its angular polarization profile always follows the polarization vector of the exciting light (Fig. 4 a). For this plane the value of $\rho$ is constant. However, the same experiments in cleaved edge geometry where the light is incident normally to the edge of the sample (perpendicular to the growth direction) give other results. The value of $\rho$ is not constant and the polarization distribution of the PL components does not follow the orientation of the polarization vector of the exciting a)
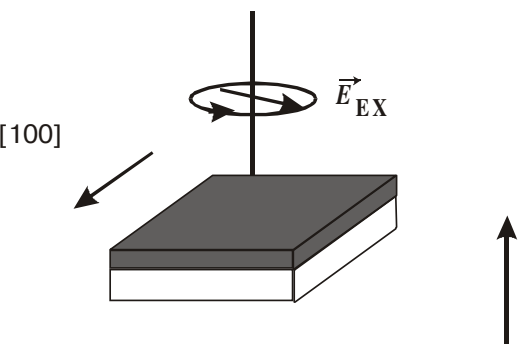

[100] b)

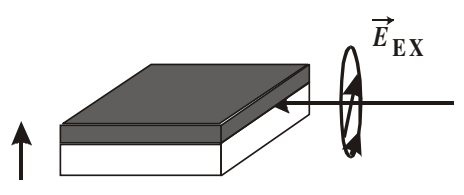

[100]
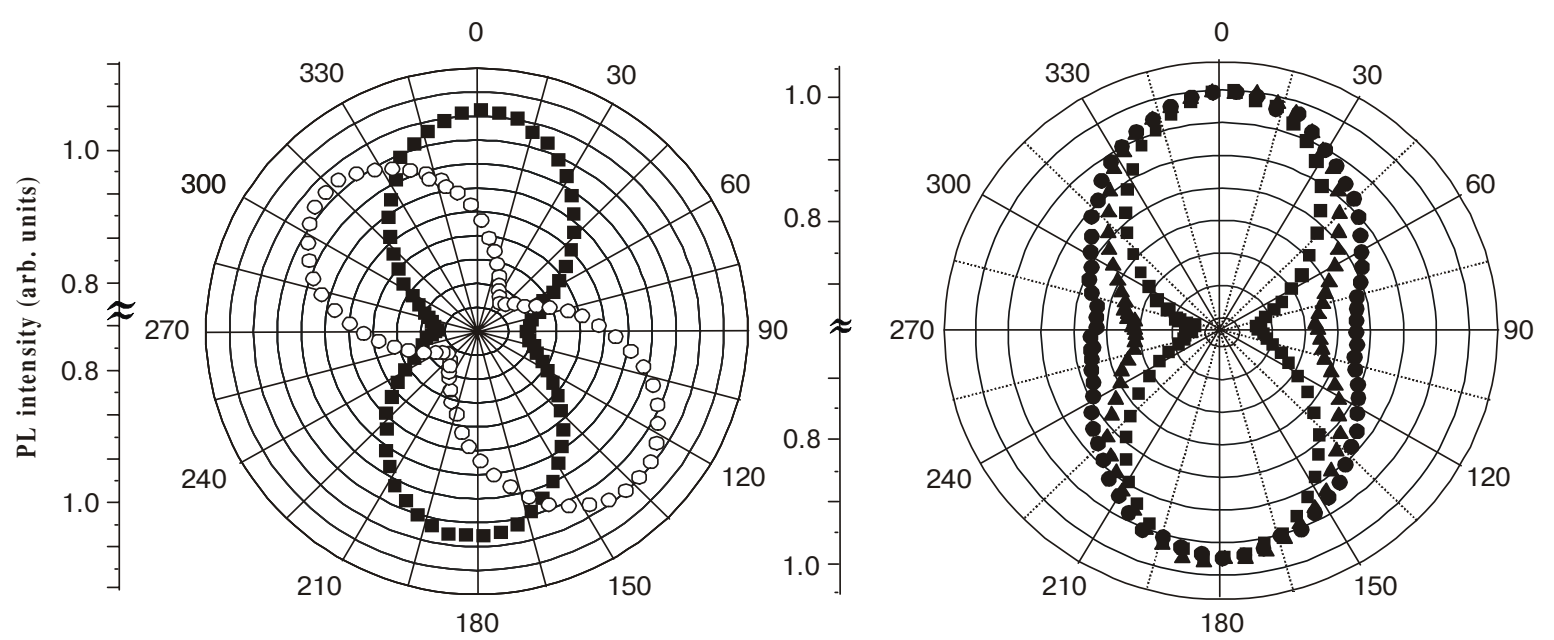

Fig. 4. a) Surface incidence: Excitation polarization is parallel (squa-res) and $-45^{\circ}$ turned (circles) to the [100] direction, b) Cleaved edge geometry: Excitation polarization is parallel (squares), $-45^{\circ}$ (triangles) and $-90^{\circ}$ turned (circles) with respect to the [100] growth direction.

Upper part: Scheme of the experimental conditions.

Lower part: Polar plot of the light intensity when the detection polaroid is turned.

$0^{\circ}$ correspond to the detection polarization parallel to the [100] direction indicated in the upper part.

$S Q O, 3(4), 2000$ 


\section{J. Diener et al.: Polarization properties of luminescence from silicon ...}

light. The PL is always partially linearly polarized parallel to the [100] growth direction of the sample, as illustrated in Fig. 4 b (for clarity all PL intensities are normalized). Within the dielectric model both geometry's reflect two limiting cases of the average alignment of the $\mathrm{NCr}$. In the edge geometry a large fraction of the $\mathrm{NCr}$ rs is oriented with their long dimension preferentially along the [100] growth direction, what is for macro-porous $\mathrm{Si}$ confirmed by TEM studies [10]. Therefore the efficiency of the excitation of $\mathrm{NCr}$ rs with their long axes parallel to the polarization direction of the exciting light will strongly depend on its orientation with respect to the [100] growth direction. As a result the PL is weaker polarized if the polarization of the exciting light is turned perpendicularly to the growth direction and a maximum of $\rho$ is achieved otherwise. However, as depicted in Fig. 4b, the main contribution to the PL comes from crystallites emitting light which is mainly polarized along their long dimensions aligned in the [100] growth direction. This preferential orientation of the PL polarization direction independent on the polarization direction of the incident light whatever it is. On the contrary, in a plane geometry the probability to excite NCrs with their long axis parallel to the orientation of polarization direction of the incident light does not depend on the direction of this polarization. Neither $\rho$ nor the intensity of the PL depend on the orientation of the polarization of the exciting light (Fig. 4a). This is a consequence of a random distribution of the NC shapes and orientations in this projection. In a framework of a dielectric model, PL polarization memory is a result of a selective excitation of a subset of crystallites with their long dimension parallel to the polarization direction of the exciting light. They will radiate light preferentially polarized in the same direction giving rise to polarized PL. Besides the asphericity and the difference in the dielectric constants of matrix and NCrs the value of should also depend strongly on the orientation selectivity of the excitation process. The excitation probability under one-photon excitation is proportional to the light intensity or square of the electric field. Multiphoton excitation processes have higher order dependencies on the field strength. Therefore, our model predicts that the value of $\rho$ should be significantly larger when the PL is excited by a two-photon excitation process what has been experimentally confirmed in [11].

\section{References}

1. L.E. Brus, Al.L. Efros and T. Itoh Spectroscopy of Isolated and Assembled Semiconductor Nanocrystals, J. Lumin, 70, ix (1996).

2. A.N. Starukhin, A.A. Lebedev, B.S. Razbirin and I.M. Kapitonova, Sov. Tech. Phys. Lett. 18, 535 (1992).

3. A.V. Andrianov, D.I. Kovalev, N.N. Zinovev and I.D Yaroshetskii, Jetp Lett. 58, 427 (1993)

4. For Example: G.E. Pikus and E.L. Ivchenko, in Excitons: Modern Problems in Condensed Matter Sciences, ed. by E.I. Rashba and M.D. Sturge, North-Holland, Amsterdam (1982).

5. F. Meier and B.P. Zakharchenya (Eds.), Optical Orientation, Modern Problems In Condensed Matter Sciences, NorthHolland Publ. Co., Amsterdam (1984)

6. P. Lavallard and R.A. Suris, Solid State Commun, 25, 267 (1995).

7. M.G. Bawend, W.L. Wilson, L. Rothberg, P.J. Carrol, T.M. Jeddju, M.L. Stegerwald and L. Brus, Phys. Rev. Lett. 65, 1623 (1990).

8. M. Chamarro, C. Gourdon and P. Lavallard, J. Lumin. 70, 222 (1996).

9. D. Kovalev, M. Ben-Chorin, J. Diener, F. Koch, Al.L. Efros, M. Rosen, M.A. Gippius and S.G. Tikhodeev, Appl. Phys. Lett. 67, 1585 (1995)

10. A.G. Cullis, L.T. Canham, and P.D.J. Calcott, J. Appl. Phys. 82, 909 (1997) and references therein.

11. J. Diener, Y. R. Shen, D. Kovalev, G. Polisski and F. Koch, Phys. Rev. B 58, 12629 (1998). 